\title{
SATELLITE ATMOSPHERES AND MAGNETOSPHERES
}

\author{
A.J. KLIORE \\ Jet Propulsion Laboratory \\ Pasadena, CA 91109 USA
}

\begin{abstract}
Galileo radio-occultation measurements show that all four of the Galilean satellites possess ionospheres. Peak ionospheric densities for the icy satellites are several thousand electrons per cubic centimeter, and the distributions are not spherically symmetric. Io's ionosphere is much denser and remarkably similar to that measured by Voyager.
\end{abstract}

Radio occultation, in which a spacecraft appears to move behind a planet or satellite as observed from Earth, affords an opportunity for the spacecraft-earth radio link to traverse the ionosphere and atmosphere of the occulting body. Interpretation of the effects of refraction by the planetary ionosphere and atmosphere allows one to infer the electron density structure in the ionosphere and the temperature-pressure profiles and absorption characteristics of the neutral atmosphere (Kliore 1972b, Fjeldbo et al., 1971).

This technique has been used very successfully to measure the characteristics of the ionospheres and atmospheres of Venus (Kliore et al., 1967; Fjeldbo et al., 1971; Kliore and Patel 1980); Mars (Kliore et al., 1965; Kliore et al., 1972a; Lindal et al., 1979); Mercury (Howard et al., 1974); Jupiter (Fjeldbo et al., 1975; Eshleman et al., 1979; Lindal et al., 1981; Hinson et al., 1997); Saturn (Kliore et al., 1980; Tyler et al., 1981; Lindal et al., 1985); Uranus (Lindal et al., 1987); Neptune (Tyler et al., 1989; Lindal 1992); Saturn's rings (Marouf and Tyler 1985); as well as Saturn's satellite Titan (Lindal et al., 1983); Neptune's Triton (Tyler et al., 1989); and Jupiter's satellites Io (Kliore et al., 1975) and Europa (Kliore et al., 1997).

Radio occultation studies of the ionospheres of Jupiter and its satellites form an important part of the Galileo Propagation Radio Science Investigation (Howard et al., 1992). To date, radio occultation measurements have been obtained with the Galileo orbiter at Europa, Ganymede, Callisto, and Io, as summarized in Table 1. The "ram angle" that is listed in Table 1 refers to the angle subtended by vectors pointing from the center of the satellite to the point on the surface at which the co-rotating magnetospheric plasma impinges normally, and to the point at which the radio beam from the spacecraft to Earth becomes tangent to the surface (the occultation point). This angle is important, because all of the Galilean satellites are immersed in Jupiter's magnetosphere, which interacts with the icy surfaces to form a tenuous atmosphere, and subsequently controls the density and shape of the ionospheres (c.f. Johnson 1990).

It is interesting to note that of the ten observations of the icy Galilean satellites listed in Table 1 , only one measurement yielded a negative result. This measurement was made at a location in the magnetospheric wake direction, where the ram angle was almost $180^{\circ}$, and where one may expect a depleted density.

The results of the Europa observations are shown in Fig. 1. In each of the three graphs comprising Fig. 1, the two electron density profiles from the entry and exit measurements of that occultation are shown along with a diagram depicting the orientation of the radio line of sight relative to the magnetospheric ram direction. The curved envelope around the satellite is a "cartoon" intended to represent the non-spherical nature of the electron density distribution resulting from its interaction with the corotating magnetospheric plasma. If the ionospheric isodensity contours are indeed shaped in such a fashion, then the longest propagation path through the ionosphere would occur when the radio line of sight is parallel to the wake direction, and the shortest when the two are normal to each other. Thus, the observed phase residual would be maximized in the former case, and minimized in the latter. This geometry assumes spherical symmetry only near the sub-ram position, and is 
TABLE 1. Geometry of Galileo Occultations by the Galilean Satellites. All occultation studies detected presence of ionosphere except for Europa 6a exit study.

\begin{tabular}{llrrrrr}
\hline Observation & Date & $\begin{array}{r}\text { Distance } \\
(\mathrm{km})\end{array}$ & $\begin{array}{r}\text { Lat. } \\
(\mathrm{deg})\end{array}$ & $\begin{array}{r}\text { W. Long. } \\
(\mathrm{deg})\end{array}$ & $\begin{array}{r}\text { SZA } \\
(\mathrm{deg})\end{array}$ & $\begin{array}{r}\text { Ram Angle } \\
(\mathrm{deg})\end{array}$ \\
\hline Europa 4 entry & $12 / 19 / 96$ & 1,600 & -2 & 346 & 95 & 76 \\
Europa 4 exit & $12 / 19 / 96$ & 4,000 & -4 & 167 & 85 & 103 \\
Europa 6a entry & $2 / 20 / 97$ & 1,500 & -24 & 281 & 86 & 11 \\
Europa 6a exit & $2 / 20 / 97$ & 4,400 & -21 & 102 & 94 & 168 \\
Europa 6b entry & $2 / 25 / 97$ & $2,777,500$ & -14 & 56 & 85 & 146 \\
Europa 6b exit & $2 / 25 / 97$ & $2,776,700$ & -14 & 236 & 95 & 34 \\
Io 6 Entry & $2 / 26 / 97$ & $3,350,400$ & 1 & 73 & 85 & 133 \\
Io 6 exit & $2 / 26 / 97$ & $3,350,200$ & 1 & 254 & 96 & 16 \\
Ganymede 8 entry & $5 / 7 / 97$ & 4,300 & 41 & 201 & 82 & 69 \\
Ganymede 8 exit & $5 / 7 / 97$ & 5,100 & 47 & 22 & 98 & 112 \\
Callisto 9 entry & $6 / 25 / 97$ & 3,900 & 2 & 163 & 82 & 107 \\
Callisto 9 exit & $6 / 25 / 97$ & 3,500 & 3 & 343 & 99 & 73 \\
\hline
\end{tabular}
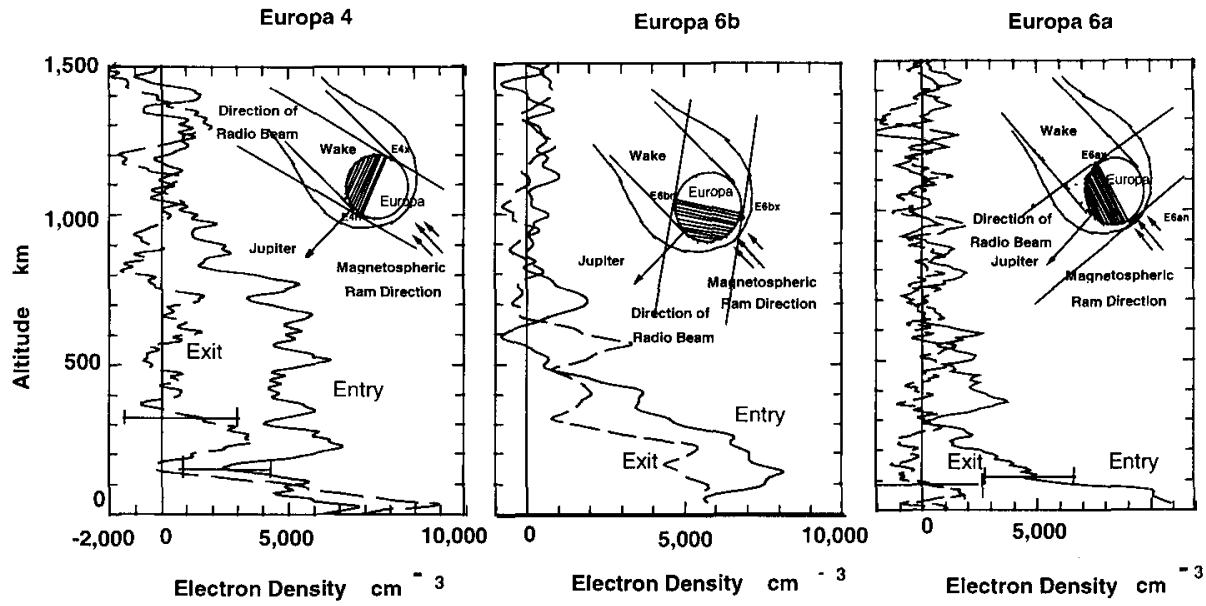

Figure 1. Europa radio occultation electron density profiles.

decidedly non-spherical elsewhere. As a result, inversion of the data using the spherical symmetry assumption may yield vertical profiles significantly different from the actual ones. This may be the case for the Europa 4 entry profile in Fig. 1.

The electron densities for Ganymede, Callisto, and Io are shown in Fig. 2. Figure 2(a) shows the results for the May 7 Ganymede occultation. From the geometry diagram it may be seen that the radio line of sight is very close to the wake direction, which may explain the "peak" at 300 $500 \mathrm{~km}$ (note that the range of the horizontal scale is half of that of Fig. 1). The Callisto results from the June 25 occultation are shown in Fig. 2(b). As in the case of the Ganymede data, the apparent peaks above $500 \mathrm{~km}$ are most likely due to effects of the occultation geometry combined with a non-spherical distribution of electron density in Callisto's ionosphere. It appears, that at least for the icy Galilean satellites, the assumption of spherical symmetry is not a very good one, and inversion methods using cylindrically symmetrical models must be developed to obtain realistic distributions of plasma in their ionospheres.

The current average electron density profiles for Europa, Ganymede, and Callisto are shown in Fig.3. With reference to Fig. 3, it should be pointed out that averaging of different measurements obtained with different occultation geometries has little meaning other than establishing a mean magnitude of electron density for each body. The large error bars for the Europa average in Fig. 3 


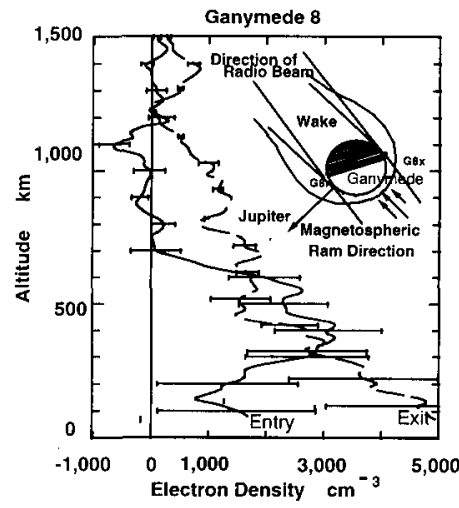

(a)

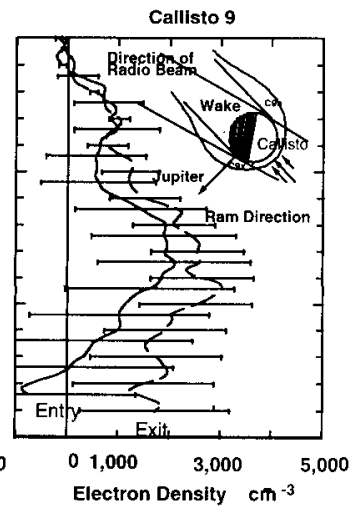

(b)

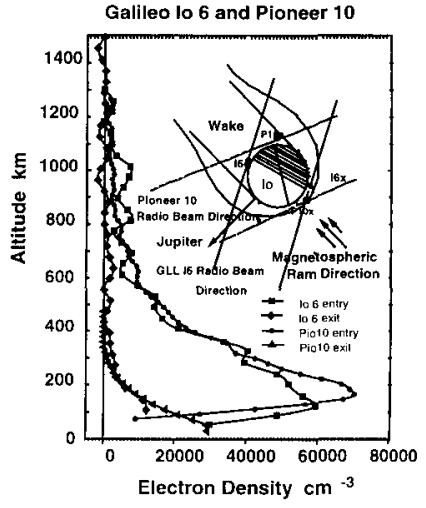

(c)

Figure 2. Electron density profiles for Ganymede, Callisto, and Io.

Europa, Ganymede, and Callisto

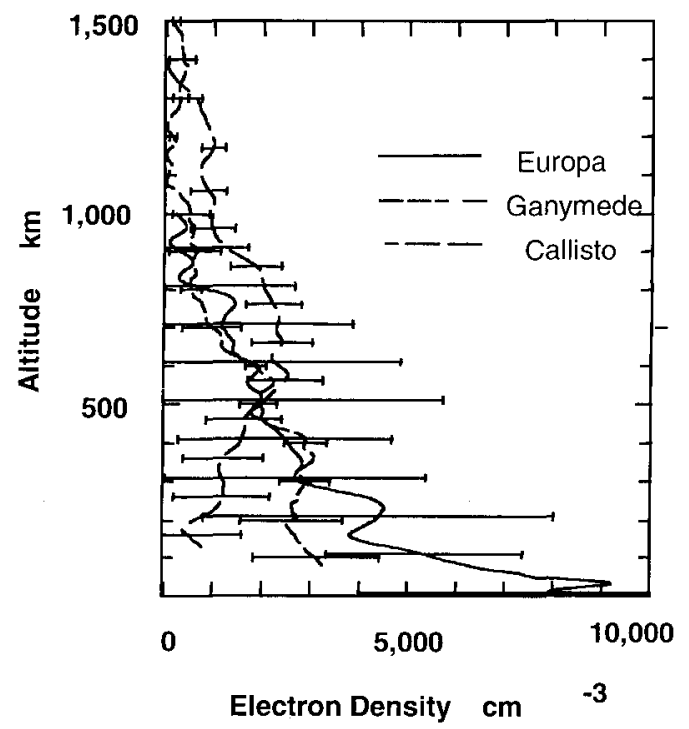

Figure 3. Averaged electron density profiles for Europa, Ganymede, and Callisto.

result from the widely different individual profiles as seen in Fig. 1. In general, the maximum electron density in the lower ionosphere is greatest for Europa (about $9,000 \mathrm{~cm}^{-3}$ ), and is lower for Ganymede (about 4,000 $\mathrm{cm}^{-3}$ ), and lower still for Callisto (about $2,000 \mathrm{~cm}^{-3}$ ).

An oxygen atmosphere on Europa was discovered with ultraviolet spectroscopy using the Goddard High Resolution Spectrograph (GHRS) on the Hubble Space Telescope (HST) in 1995 (Hall et al., 1995; Hunten 1995). In 1996, HST observations were again made on Europa, and a similas oxygen atmosphere was observed on Ganymede (Hall 1997). In addition, the Galiloo UV spectrometer detected excess Lyman- $\alpha$ emission from a hydrogen exosphere on Ganymede (Barth et al., 1997). All of these observations are consistent with the existence of tenuous oxygen atmospheres on Europa, Ganymede, and Callisto, produced by sputtering of surface water ice by energetic particles from Jupiter's magnetosphere (Johnson 1990). The $\mathrm{O}_{2}$ column densities inferred from the HST observations are 2.4 to $14 \times 10^{14} \mathrm{~cm}^{-2}$ for Europa, and 1 to $10 \times 10^{14} \mathrm{~cm}^{-2}$ for Ganymede (Hall et al., 
1997). From the radio occultation electron density measurements at Europa, assuming $\mathrm{O}_{2}^{+}$to be the principal ion, the surface density of $\mathrm{O}_{2}$ can be inferred to be $10^{7}$ to $10^{8} \mathrm{~cm}^{-3}$, and the corresponding vertical column density with a neutral atmosphere scale height of $120 \mathrm{~km}$, and taking into account both photoionization an electron impact ionization, is 2.4 to $24 \times 10^{14}$ (Kliore et al., 1997). Such a density is consistent with the HST observations, as is the reduced density observed on Ganymede. The ionosphere of Io was discovered by the radio occultation of Pioneer 10 (Kliore et al., 1975), and it and its underlying atmosphere have been the subject of numerous studies and observations since then (Johnson and Matson 1976; Kumar and Hunten 1982, Kumar 1985; Lellouch et al., 1990, 1992). The atmosphere of Io is fundamentally different from those of the icy Galilean satellites, consisting of $\mathrm{SO}_{2}$ ejected from volcanoes and possibly sulphur and its compounds sputtered from its surface. As can be seen from Fig. 2(c), its ionosphere is also quite different. It is about $10-100$ times denser, depending on the location, and has a well-defined peak. The geometry diagram of Fig. 2(c) shows that although the direction of the radio beam relative to the direction to Jupiter is quite different for the Galileo 16 and Pioneer 10 occultations, the location of the entry and exit points relative to the magnetospheric ram direction is quite similar. It is therefore not surprising that the observed electron density profiles are very similar. What is somewhat surprising is the implication that the structure of the atmosphere has not changed significantly over some 23 years, indicating a stable equilibrium between source and loss mechanisms. During the close encounters of the Galileo spacecraft with Jupiter's Galilean satellites, the Galileo Magnetometer instrument was used to investigate the magnetic fields in the environs of Io, Europa, Ganymede, and Callisto. Ganymede was discovered to have an intrinsic magnetic field strong enough to stand off Jupiter's magnetosphere and form its own magnetospheric environment (Kivelson et al., 1996a, 1997b). The close fly-by of Io yielded unique magnetic signatures which may indicate the presence of a magnetic field, but its interpretation is complicated by Io's interaction with its torus (Kivelson et al., 1996b, 1996c; Khurana et al., 1997b). Europa provided a signature which indicates that it also has an intrinsic magnetic field (Kivelson et al., 1997a). Of the Galilean satellites, only Callisto was conclusively observed not to have a magnetic field (Khurana et al., 1997a).

\section{Acknowledgements}

The author wishes to acknowledge the contributions of the members of the Galileo Propagation Radio Science Team: D.P. Hinson, F.M. Flasar, R. Woo, V.R. Eshleman, and H.T. Howard, the Team Leader. The Radio Science Support Team at JPL, especially R. Herrera, S. Asmar, D. Chong, P. Eshe, P. Priest, A. Anabtawi, T. Rebold, S. Abbate, and J. Caetta provided essential support in the acquisition and processing of the data. The Gallieo Project Team, especially the Navigation Team under W.E. Kirhofer, J. Johanessen, and J. Pojman, provided the resources and trajectory information necessary to interpret the data. M. Kivelson, P.I. of the Galileo Magnetometer experiment, as well as K. Khurana and C. Polanskey, kindly provided material on their discoveries on the magnetic fields of the satellites. This work was performed at the Jet Propulsion Laboratory, California Institute of Technology, under NASA contracts.

\section{References}

Barth, C.A. et al. (1997) Geophys. Res. Lett., in press.

Eshleman, V. et al. (1979) Science, 204, pp. 976-978.

Fjeldbo, G. et al. (1971) Astron. J., 76, p. 123.

Fjeldbo, G. et al. (1975) Astron. and Astrophys., 39, pp. 91-96.

Hall, D.T. et al. (1997) Astrophys. J., in press.

Hall., D.T. et al. (1995) Nature, 373, p. 677.

Hinson, D.P. et al. (1997) Geophys. Res. Lett., in press.

Howard, H.T. et al. (1974) Science, 185, pp. 179-180.

Howard, H.T. et al. (1992) Space Sci. Rev., 60, pp. 565-590.

Hunten, D.M. (1995) Nature, 373, p. 654.

Johnson, R.E. (1990) Energetic Charged Particle Interactions With Atmospheres and Surfaces, Springer Verlag, New York.

Johnson, T.V. and D.L. Matson (1976) Geophys. Res. Lett., 3, pp. 293-296.

Khurana, K.K. et al. (1997a) Nature, 387, p. 262.

Khurana, K.K. et al. (1997b) Geophys. Res. Lett., in press.

Kivelson, M.G. et al. (1996a) Nature, 384, p. 537.

Kivelson, M.G. et al. (1996b) Science, 274, p. 396.

Kivelson, M.G. et al. (1996c) Science, 273, p. 337. 
Kivelson, M.G. et al. (1997a) Science, 276, p. 1239.

Kivelson, M.G. et al. (1997b) Geophys. Res. Lett., in press.

Kliore, A.J. et al. (1965) Science, 149, pp. 1243-1248.

Kliore, A.J. et al. (1967) Science, 158, pp. 1683-1688.

Kliore, A.J. et al. (1972a) Icarus, 12, pp. 484-516.

Kliore, A.J. (1972b) In: The Mathemathics of Profile Inversion, L. Colin, (Ed.), pp. 3-2, NASA Ames Research Center, Moffett Field, CA.

Kliore, A.J. et al. (1975) Icarus, 24, pp. 407-410.

Kliore, A.J. et al. (1980) J. Geophys. Res., 85, pp. 5857-5870.

Kliore, A.J. and I.R. Patel (1980) J. Geophys. Res., 85, pp. 7957-7962.

Kliore, A.J. et al. (1997) Science, 277, p. 355.

Kumar, S. and D.M. Hunten (1982) In: Satellites of Jupiter, D. Morrison (Ed.), pp. 782-806, University of Arizona Press, Tucson, Arizona.

Kumar, S. (1985) Icarus, 61, pp. 101-123.

Lellouch, E. et al. (1990) Nature, 346, pp. 639-641.

Lellouch, E, et al. (1992) Icarus, 98, pp. 271-295.

Lindal, G. et al. (1981) J. Geophys. Res., 86, pp. 8721-8727.

Lindal, G.F. et al. (1979) J. Geophys. Res., 84, pp. 8443-8456.

Lindal, G.F. et al. (1983) Icarus, 53, pp. 348-363.

Lindal, G.F. et al. (1985) Astron. J., 90, pp. 1136-1146.

Lindal, G.F. et al. (1987) J. Geophys. Res., 92, pp. 149870-15001.

Lindal, G.F. (1992) Astron. J., 103, pp. 967-982.

Marouf, E.A. and G.L. Tyler (1985) Adv. Space Res., 5, pp. 117-120.

Tyler, G.L. et al. (1981) Science, 212, pp. 201-206.

Tyler, G.L. et al. (1989) Science, 246, pp. 1466-1473. 